\title{
Lidil
}

Revue de linguistique et de didactique des langues

\section{Le rôle des connaissances morphographiques dans l'acquisition de l'orthographe aux cycles 2 et 3}

\section{Christine Sanguin-Bruckert et Jean-Pierre Bruckert}

\section{(2) OpenEdition}

\section{Journals}

Édition électronique

URL : http://journals.openedition.org/lidil/893

DOI : $10.4000 /$ lidil. 893

ISSN : 1960-6052

\section{Éditeur}

UGA Éditions/Université Grenoble Alpes

\section{Édition imprimée}

Date de publication : 1 décembre 2004

Pagination : 147-167

ISBN : 2-914176-11-2

ISSN : 1146-6480

\section{Référence électronique}

Christine Sanguin-Bruckert et Jean-Pierre Bruckert, « Le rôle des connaissances morphographiques dans l'acquisition de l'orthographe aux cycles 2 et 3 », Lidil [En ligne], 30 | 2004, mis en ligne le 29 janvier 2008, consulté le 01 mai 2019. URL : http://journals.openedition.org/lidil/893 ; DOI : 10.4000/ lidil.893 
LE RÔLE DES CONNAISSANCES MORPHOGRAPHIQUES DANS L'ACQUISITION DE L'ORTHOGRAPHE AUX CYCLES 2 ET 3

Christine SANGUIN-BRUCKERT * Jean-Pierre BRUCKERT **

Les deux composantes du traitement de la langue écrite, lire et orthographier, sont en général considérées comme des activités «en miroir», c'est-à-dire régies par les mêmes processus: d'une part les procédures alphabétiques, c'est-à-dire l'application des correspondances phono-graphémiques, et d'autre part les procédures lexicales avec la récupération automatique des représentations orthographiques dans le lexique mental (Sanguin-Bruckert et Bruckert, 2001). Les recherches en psychologie cognitive traitant des compétences en orthographe sont beaucoup moins nombreuses que celles traitant de la lecture, et si elles confirment l'utilisation de processus communs, elles font apparaitre aussi les spécificités de chacune de ces activités (Bosman et Van Orden, 1997).

\section{Bref rappel sur les processus}

\section{Orthographe vs lecture}

De nombreux auteurs (Perfetti, 1997; Mousty et Alégria, 1999; Gombert, Bryant et Warrick, 1997...) soulignent

* Laboratoire EA1588 «Processus cognitifs et conduites interactives», Département de psychologie, Université Paris 10-Nanterre.

<chbruckert@aol.com> 
notamment que, dans ces deux activités, les différents processus de récupération des mots dans le lexique mental ne sont pas identiques. En effet la restitution d'un mot en lecture à partir du stimulus écrit peut être assimilée à une procédure de recherche en mémoire par reconnaissance ${ }^{1}$. Il suffit d'associer le percept du stimulus écrit avec la représentation mentale lexicale la plus probable et la plus pertinente en fonction du contexte textuel et situationnel et des propriétés orthographiques du mot, même si cette représentation est imprécise ou incomplète. En orthographe, la récupération dans le lexique peut être assimilée à une procédure de rappel ; l'indice disponible est le plus souvent la forme phonologique qui doit permettre de retrouver la forme écrite du mot. L'analyse de ce mot doit alors être complète (analyse de tous les segments) pour que la récupération de la forme orthographique soit correcte. On sait maintenant que l'identification des mots chez le lecteur expert fait appel à des procédures, assemblage ou adressage, totalement automatiques, alors que la scription exige très souvent même chez le lecteur accompli une recherche active, voire laborieuse, et nécessite le recours à des indices complémentaires. Cette remarque explique pourquoi l'orthographe est une activité inévitablement moins automatisée que la lecture.

\section{Le système orthographique du français}

Un autre point à soulever concerne le système d'écriture dans lequel se situent ces deux activités. Si elles sont toutes deux dépendantes de ce système, l'orthographe semble plus sensible encore aux caractéristiques propres à chacune des langues. Il existe deux grands types d'orthographes: celles que l'on appelle «transparentes», c'est-à-dire essentiellement régies par des correspondances graphèmes/phonèmes régu-

1. Il y a deux formes de récupération en mémoire d'un matériel précédemment appris. Dans la «reconnaissance», ce matériel est présenté à nouveau et doit être identifié comme déjà connu; dans le «rappel», il doit être évoqué à partir d'indices plus ou moins importants, ce qui est plus difficile. 
lières, stables et univoques (finnois, italien...) et celles dites « opaques» ou "profondes» qui, tout en observant aussi le principe des correspondances phono-graphémiques, reflètent également les composantes morpho-syntaxiques de la langue. Le système français (avec l'anglais dans une moindre mesure) en est un bon exemple. Catach $(1980,1988)$ a proposé un modèle "plurisystémique» pour décrire l'orthographe française ; même s'il peut être critiqué quant à son apparente simplicité (Millet, 1996), il a l'avantage de fournir un modèle fonctionnel utile dans la réflexion menée sur l'apprentissage de l'orthographe. Dans ce plurisystème, les marques morphogrammiques sont soit grammaticales (le $« s »$ du pluriel), soit lexicales (les lettres muettes marquant les relations entre formes fléchies: «grand»/ «grande», ou dérivées: «bord»/ «bordure») et ne peuvent être obtenues par une simple transcription phonographique. Elles sont nécessairement le résultat soit d'une procédure lexicale (récupération d'un mot connu à partir d'une représentation orthographique stockée en mémoire), soit de la mise en œuvre de connaissances syntaxiques (règles de grammaire), contextuelles orthographiques (la substitution de « $n$ » par «m» devant un « $b$ » ou un «p»), étymologiques (l'accent circonflexe sur le «a» dans château qui remplace le «s» de «castel») ou bien de connaissances sur les liens morphologiques.

\section{Les modèles du développement}

De nombreux modèles d'acquisition de l'orthographe ont été proposés (Henderson, 1985; Ehri, 1986; Ferreiro, GomezPalacio et al., 1988; Sprenger-Charolles et Casalis, 1996; Seymour, 1997) dont on peut décrire de grandes étapes communes. Au cours des années pré-scolaires, les enfants qui découvrent l'écrit ne le considèrent pas encore comme une codification du son (principe alphabétique) mais comme une codification du sens (les signes graphiques sont alors considérés soit comme des pictogrammes, soit comme des idéogrammes). Ensuite, ils comprennent que le lien entre lettres et sons est arbitraire mais ils l'appliquent aussi bien aux syllabes orales qu'aux phonèmes; ainsi observe-t-on souvent l'utilisation du nom des lettres pour représenter les syllabes: «terre» 
s'écrira «TR», « céder» sera noté « $C D$ ». Avec l'introduction plus systématique du code grapho-phonologique à l'entrée au $\mathrm{CP}$, l'enfant va pouvoir commencer à élaborer des représentations complètes tant phonologiques qu'orthographiques des mots; ses tentatives de scription sont guidées en grande partie par des procédures phonographiques (Chevrot, 1996) mais en utilisant des correspondances acquises très simplifiées (notion de "graphonèmes dominants" proposée par Alégria et Mousty, 1997) ${ }^{2}$. Enfin, les contraintes orthographiques conventionnelles et les marques syntaxiques et morphologiques sont prises en compte pour conduire l'enfant à une orthographe correcte (étape morphémique).

Recherches actuelles sur le rôle de la morphologie dans l'acquisition de l'orthographe

Les travaux en français traitant du rôle de la morphologie dans l'écrit sont récents, peu nombreux, et les modèles concernant l'apprentissage de l'orthographe ne s'accordent ni sur l'âge ni sur l'importance de l'utilisation de ces connaissances dans cette acquisition. Rappelons que dans les modèles d'acquisition de l'orthographe proposés par Henderson (1985), Erhi (1986) ou Ferreiro, Gomez-Palacio et al. (1988), le recours à la morphologie est la dernière étape de cet apprentissage, après la maitrise du code phonographique. Totereau, Thévenin et Fayol (1997) et Thévenin, Totereau, Fayol et Jarousse (1999) montrent que l'acquisition de la morphologie écrite du nombre s'effectue en plusieurs étapes qui conduisent progressivement à une plus grande automatisation correspondant à l'activation en mémoire de la flexion correspondant à telle ou telle configuration singulière ou plurielle. Mais cette automatisation ne serait que partielle (même chez le scripteur expert) parce que sensible aux surcharges cognitives imposées par des tâches additives ou distractives,

2. Alégria et Mousty définissent le terme "graphonème» comme la «traduction phonème-graphème», c'est-à-dire la relation qui unit les unités graphiques aux phonèmes correspondants (p. 167). 
d'où la réapparition d'erreurs d'accord dans des situations complexes de production écrite (plus d'erreurs par exemple dans l'accord du sujet avec le verbe quand ce dernier est séparé de son sujet par divers éléments : «Les maisons du petit village qui dormait sur la colline *était/étaient construites en bois »).

Il semble néanmoins difficile de transférer les processus d'acquisition et d'automatisation des règles d'accord en nombre à ceux qui conduisent aux accords en genre. A l'oral, la marque du féminin (ajout de «e») est plus fréquemment prononçable que les marques du pluriel ( $s \mathrm{~s}$ » ou « $\mathrm{x} \gg$ muets) et donc plus aisément repérable lors du passage à l'écrit. Cette caractéristique pourrait être un indice à la récupération de la forme masculine (le masculin "petit» par exemple renvoie à la forme féminine «petite», ce qui aide à retrouver la lettre muette «t»). Alégria et Mousty $(1994,1997)$ et Mousty et Alégria (1999), dans une recherche étudiant le rôle des connaissances de l'orthographe des formes féminines pour retrouver celle des formes masculines, montrent que la présence de mots inducteurs au féminin favorise la récupération des lettres terminales muettes des mots cibles masculins. Mais ils n'obtiennent pas de résultats significatifs concernant l'utilisation spontanée de ces connaissances dans des tâches de dictée classique (sans indice inducteur). Nous reprendrons ce point dans la discussion des résultats.

Treiman et Cassar (1997), contrairement aux modèles classiques cités plus haut, mettent en évidence que les jeunes enfants, avant même l'apprentissage de l'écrit, connaissent et respectent les règles d'espacement entre les mots, d'orientation de l'écriture, de compatibilité des séquences de lettres à l'intérieur des mots (en anglais «ck» ou «ee»), de positionnement des lettres dans le mot (en français ou en anglais le doublement des lettres «ll» ou «nn» n'apparait qu'en milieu ou fin de mot, jamais en position initiale). Ces auteurs observent également que chez les jeunes apprentis orthographieurs les confusions orthographiques entre « $t$ » et « $d$ » à l'intérieur de mots comportant des battements étaient moins nombreuses dans le cas des mots comportant deux morphèmes comme «durty» que pour ceux à structure monomorphèmique comme «duty». Ils concluent que si ces jeunes élèves utili- 
saient uniquement les informations phonologiques, ils devraient présenter autant d'erreurs pour les deux types de mots ; l'observation du contraire va dans le sens d'une utilisation de la relation sémantique entre la racine et le mot dérivé et de la prise de conscience que cette relation est marquée dans l'orthographe.

\section{Problématique}

En nous appuyant sur ces derniers auteurs, nous allons tenter de montrer que les connaissances morphologiques, que l'on peut considérer comme des connaissances métalinguistiques, c'est-à-dire des connaissances concernant la structure et le fonctionnement de la langue, se construisent en même temps que toutes les autres connaissances de ce type et que, comme toute activité réflexive, elles interviennent dès le début de l'apprentissage du langage écrit (Sanguin-Bruckert et Bruckert, 2003). La majorité des études en Psychologie de la lecture s'accordent pour considérer les capacités métalinguistiques en général, et métaphonologiques en particulier, comme les meilleurs prédicteurs de l'apprentissage de la lecture; elles soulignent également l'intervention de ces capacités dans l'acquisition de l'orthographe (Sprenger-Charolles et Casalis, 1996; Mousty et Alegria, 1996). On peut donc penser que la compréhension des liens flexionnels et dérivationnels entre les mots se développe parallèlement à celle qui concerne les autres caractéristiques de la langue.

Nous avons mené dans ce sens deux études, la première portant sur le rôle des connaissances morphologiques relatives au genre, et la seconde sur les capacités de transfert des propriétés orthographiques d'un mot à ses dérivés morphologiques. La question qui sera débattue à l'issue de nos résultats sera celle de la conscience de ces connaissances métamorphologiques, de leur caractère implicite ou explicite.

\section{Expérience I}

Nous avons vu que l'orthographe en français rend compte de la morphologie de la langue, en particulier par l'existence de 
lettres muettes en position terminale qui indiquent le lien entre les formes fléchies des mots (grand/grande) ou entre les mots simples et les formes dérivées (chat/chaton). Nous nous sommes donc demandé si l'apprenti orthographieur était capable d'utiliser les connaissances qu'il possède des formes féminines des substantifs et adjectifs pour en inférer les lettres muettes terminales des formes masculines, et à quel moment il acquérait ces connaissances pour y avoir recours spontanément.

\section{Population}

L'expérience a été menée au mois de mai, au sein des classes. Elle porte sur 3 niveaux scolaires: 38 enfants de CP ( 3 classes, 2 écoles), 36 enfants de CE1 ( 3 classes, 2 écoles), 48 enfants de CE2 ( 3 classes, 2 écoles).

\section{Les épreuves}

La tâche consiste à compléter l'orthographe lacunaire de mots comportant une lettre muette en position finale et insérés dans des phrases présentées à la fois par écrit et oralement. Tous les mots ont été sélectionnés du fait de leur présence dans les manuels de lecture de CP ou bien encore dans des ouvrages d'enfant destinés à cet âge ${ }^{3}$; cette procédure permet raisonnablement de les considérer comme équivalents quant à leur fréquence à l'écrit. Cette tâche est composée de 3 épreuves. Les deux premières épreuves comportent 2 types de mots masculins se terminant par une lettre muette: $50 \%$ d'entre eux possèdent une forme fléchie (avocat/avocate), $50 \%$ n'en possèdent pas (escargot). Nous cherchons à savoir si l'existence d'une forme féminine peut améliorer les performances orthographiques quant à la récupération de la lettre muette au masculin. Si tel est le cas, nous pouvons alors inférer que les enfants se sont appuyés sur leurs connaissances des liens morphologiques pour produire une orthographe correcte.

3. De type «Belles Histoires» ou «J'aime Lire» (Bayard Presses Jeune), Folio Benjamin, Folio Junior... 
La première épreuve de dictée classique met en œuvre une recherche en mémoire de type rappel (elle sera d'ailleurs notée «rappel de mots» ultérieurement); elle se présente sous cette forme:

- Le sac de ma mère est lou.

La seconde est une épreuve à choix multiples présentée ainsi :

- C'est le petit de la bique, c'est le. biquet

biquè biquai

Elle fait appel à un processus de reconnaissance (et sera notée «reconnaissance de mots»).

La troisième épreuve de même type que la $1^{\text {re }}$ introduit un indice supplémentaire en faisant apparaitre la forme féminine au sein même de la phrase (notée «inductions de mots»):

- Ma sœur est blonde et mon frère est blo_ aussi

Cette nouvelle épreuve, par construction, ne comporte pas de mots sans forme fléchie. Elle a pour objectif de montrer que les enfants les plus jeunes, s'ils s'avèrent encore incapables de trouver par eux-même les liens entre masculin et féminin, peuvent les utiliser pour retrouver la lettre muette lorsque ces liens leur sont donnés explicitement.

Le matériel comprend 2 types de mots: adjectifs et substantifs, 5 types de lettres muettes terminales (s, t, d, er, et) et 12 items par série (donc 36 à 24 par épreuve, puisque la $3^{e}$ épreuve par construction ne comporte pas de substantifs sans forme fléchie).

Afin de contrôler l'effet d'apprentissage, les mots-cibles des deux premières épreuves (proposées lors de la même passation) sont différents mais jugés équivalents quant à leur fréquence, leur complexité sémantique et le type de lettre muette terminale. Quant à la $3^{\mathrm{e}}$ épreuve, elle reprend les mots-cibles de l'épreuve de dictée classique, et a donc été passée après un délai de trois semaines.

\section{Hypothèses}

H1. Les performances en orthographe augmentent avec le niveau scolaire ${ }^{4}$.

H2. Les performances en orthographe dépendent du type

4. Cette hypothèse apparemment triviale nous permet de valider notre matériel expérimental. 
d'épreuves proposées; l'épreuve de dictée classique qui ne donne qu'une information phonologique sur l'orthographe du mot sera plus difficile que celles qui proposent des indices soit sous la forme du stimulus écrit (reconnaissance), soit sous la forme morphologique en amorce (forme fléchie).

H3. Les enfants utilisent précocement les connaissances qu'ils possèdent sur les liens entre forme féminine et forme masculine des mots pour retrouver les lettres muettes de la forme masculine; ces connaissances sont partiellement présentes dès le début de l'apprentissage; elles s'enrichissent et se diversifient avec la scolarité.

\section{Plan d'expérience}

Nous avons 3 groupes de sujets $(\underline{S})$ indépendants correspondant aux 3 niveaux scolaires $(\mathrm{N})$, qui participent aux 3 épreuves $(E)$, avec les deux types de mots $(M)$ et pour lesquels il existe ou non une forme fléchie s'appuyant sur la lettre muette $(F)$, soit: $\underline{\mathrm{S}}<\mathrm{N}_{3}>* \mathrm{E}_{3} * \mathrm{M}_{2} * \mathrm{~F}_{2}$.

\section{Résultats}

En préambule, nous devons préciser que nous n'avons pas trouvé de différences significatives, quelle que soit la tâche, entre les performances obtenues avec les substantifs pouvant prendre une forme féminine à partir de la lettre muette et les adjectifs sélectionnés.

Effet du niveau scolaire et du type de tâche

Afin de comparer les différents niveaux scolaires sur des épreuves équivalentes, nous n'avons pris en compte que les résultats aux items concernant les mots (substantifs et adjectifs) comportant une forme féminine (F) (score max.: 24) puisque dans la $3^{\mathrm{e}}$ épreuve nous proposons en amorce les mots sous leur forme féminine, éliminant ainsi ceux qui n'ont pas de féminin. 


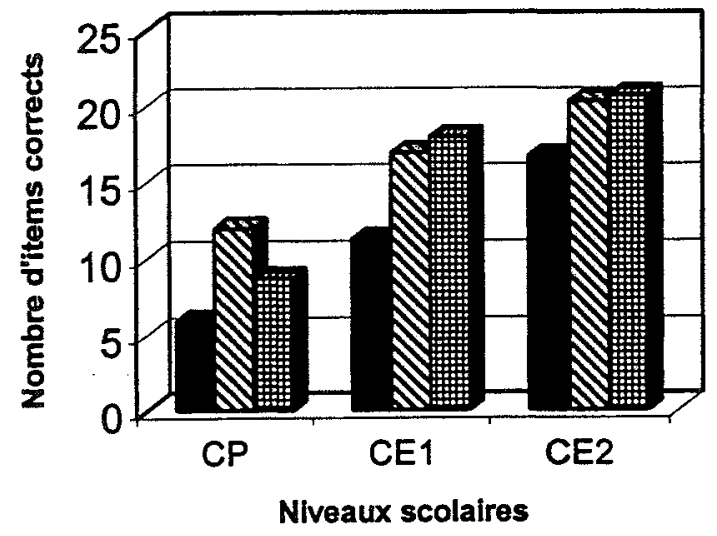

\section{rap mots $F \quad$ reco mots $F$ Induction}

Figure 1 - Effet du type de tâche et du niveau scolaire sur le nombre d'items correctement orthographiés en ce qui concerne la présence des lettres muettes.

Nous voyons que les scores augmentent avec l'âge mais surtout qu'ils dépendent du type de tâche. Les résultats pour la dictée classique (rappel) sont significativement inférieurs à ceux des autres épreuves et ceci pour tous les niveaux. L'analyse de variance montre un effet très significatif de l'âge (la probabilité p que ce résultat soit dû au hasard est inférieure à $1 / 100000^{e}$, soit $\left.\mathrm{p}<0,00001\right)$ et du type d'épreuve $(\mathrm{p}<0,00001)$, mais indique aussi un effet d'interaction des 2 facteurs $(p<0,005)$ que l'on peut imputer aux résultats obtenus en CP. En effet, on observe que dans cette classe l'épreuve de reconnaissance est mieux réussie que celle d'orthographe indicée, alors que l'on constate que la différence s'estompe pour les classes supérieures (elle n'est pas significative).

On peut interpréter ces résultats par la difficuité que présente encore pour les jeunes enfants l'utilisation des liens féminin/masculin, alors qu'ils possèdent déjà des représentations orthographiques des mots les plus fréquents, mots qu'ils peuvent récupérer directement par la voie lexicale plus automatique et favorisée par la tâche de reconnaissance. Mais il est à noter malgré tout qu'un certain nombre d'enfants sont 
déjà capables d'inférer les lettres muettes à partir des indices morphologiques qui leur sont fournis.

- Les hypothèses 1 et 2 sont confirmées.

Effet de l'existence de formes fléchies, en fonction du niveau et du type de tâche

Un deuxième type de résultats précise les premiers. Nous avons étudié les différences de performance uniquement entre les substantifs présentant une forme féminine $(F)$ et ceux qui n'en possèdent pas (NF) (puisque nos adjectifs peuvent toujours prendre la marque du féminin).

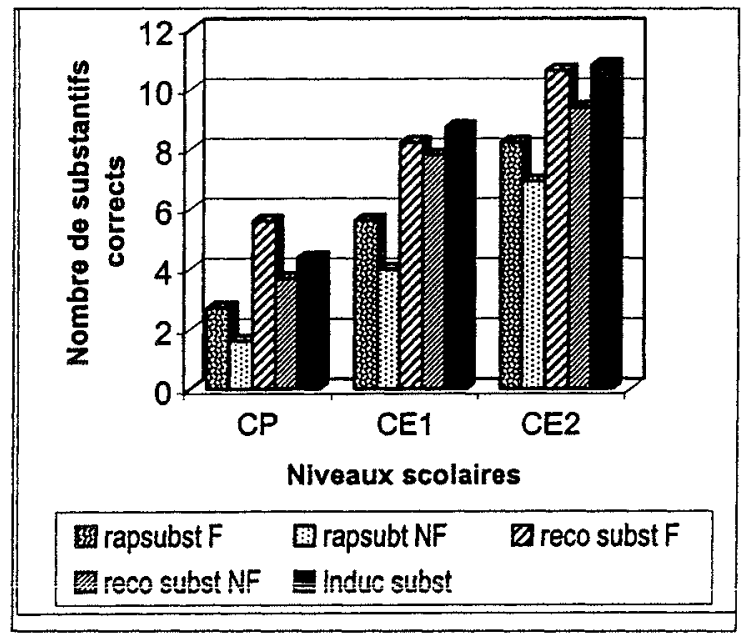

Figure 2 -Effet de l'existence des marques féminines, du niveau scolaire et du type de tâche, sur le nombre de substantifs correctement orthographiés en ce qui concerne la présence des lettres muettes

Nous observons que pour tous les niveaux les substantifs présentant une forme féminine sont mieux orthographiés que les autres ${ }^{5}$. L'analyse de variance montre un effet très significatif de l'âge $(p<0,00001)$, du type d'épreuve $(p<0,00001)$, mais

5. Le test statistique du t de Student indique que les différences entre les scores corrects des formes fléchies et non-fléchies sont significatives à $\mathrm{p}<0,01$, sauf pour l'épreuve de reconnaissance au CEl. 
aussi un effet d'interaction significatif des 2 facteurs ( $p$ $<0,01$ ), qui s'explique surtout par le fait qu'en situation de reconnaissance les différences diminuent avec le niveau scolaire. Ceci peut alors s'expliquer par l'utilisation beaucoup plus massive de la voie lexicale au fur et à mesure des apprentissages scolaires. En revanche, en ce qui concerne l'épreuve de dictée classique, on peut conclure à une aide non négligeable des connaissances que possèdent les enfants sur la morphologie des formes fléchies pour résoudre leurs problèmes d'orthographe des lettres muettes, et ce dès la première année d'école primaire.

- L'hypothèse 3 est donc confirmée.

Il est intéressant de remarquer que ces résultats ne sont pas conformes à ceux recueillis par Alégria et Mousty (1994) et Mousty et Alégria (1999) sur des enfants d'âges équivalents ; en effet, ils ne trouvent pas de différences significatives entre mots ayant un féminin et ceux n'en possédant pas. Dans cette recherche, les auteurs ont utilisé des mots fréquents et d'autres non fréquents; peut-être est-ce cette différence avec notre propre travail (où les mots étaient jugés comme plutôt fréquents) qui peut expliquer cette divergence de résultats.

\section{Expérience /l}

Nous venons de voir que les enfants possèdent des connaissances précoces sur les liens de flexion morphologiques et sont capables de les utiliser pour inférer les lettres finales muettes des substantifs et adjectifs; mais retrouve-t-on ces compétences dans le cas des dérivations? Pour répondre à cette question, nous avons mené une $2^{\mathrm{c}}$ recherche comportant 3 épreuves.

\section{Épreuve 1}

Dispositif expérimental

La première épreuve comporte deux phases:

- une phase d'apprentissage: tous les sujets ont appris préa- 
lablement, en classe et avec leur professeur (à raison de 2 séances par semaine pendant 4 semaines), 20 mots référents possédant tous une difficulté orthographique particulière (doublement de consonne; choix entre plusieurs phonogrammes, par exemple «en» ou «an»; lettre muette à l'intérieur ou au début du mot comme dans «thé » ou «hache»);

- une phase expérimentale: les élèves sont soumis à une épreuve d'orthographe classique: retrouver l'orthographe de mots insérés dans des phrases simples, présentées écrites et dictées par l'expérimentateur (« exercice à trous»). Trois types de mots, appariés sur leur fréquence, étaient proposés :

- dérivés: 20 mots dérivés des mots référents, par exemple « déterrer» pour «terre»;

- non dérivés : 20 mots présentant la même difficulté orthographique mais n'ayant aucun lien sémantique avec les mots référents appris, par exemple «guerrier»/《terre»;

- orthographiquement simples: 20 mots ne présentant pas cette difficulté orthographique et se transcrivant à l'aide des phonogrammes les plus fréquents (un $« \mathrm{r}$ » unique plus fréquent que « $2 r$ » comme dans «parier», « $f$ » plus fréquent que «ph» comme dans «sofa»). Dans la suite de l'article, pour alléger l'écriture, nous les nommerons «simples».

Cette épreuve a été simplifiée pour les élèves de $\mathrm{CP}$ en ne conservant que les 10 mots référents les plus fréquents et les plus faciles du point de vue de l'orthographe.

\section{Population}

Cette seconde étude s'intéresse à l'ensemble des classes du primaire: 268 sujets dans 3 écoles et 5 niveaux scolaires: CP (52 sujets), CE1 (52), CE2 (55), CM1 (63), CM2 (46). Les épreuves ont été proposées en fin d'année scolaire et toujours dans les classes.

\section{Hypothèse}

H4. Les connaissances des difficultés orthographiques d'un mot familier pourront être généralisées aux mots dérivés de la même famille lexicale, et ce dès le début de l'apprentissage de l'écrit. 


\section{Plan d'expérience}

Nous avons 5 groupes indépendants correspondant aux 5 niveaux scolaires $(\mathrm{N})$, et tous les élèves sont confrontés aux 3 types de mots (M), soit: $\underline{\mathrm{S}}<\mathrm{N}_{5}>* \mathrm{M}_{3}$.

\section{Résultats}

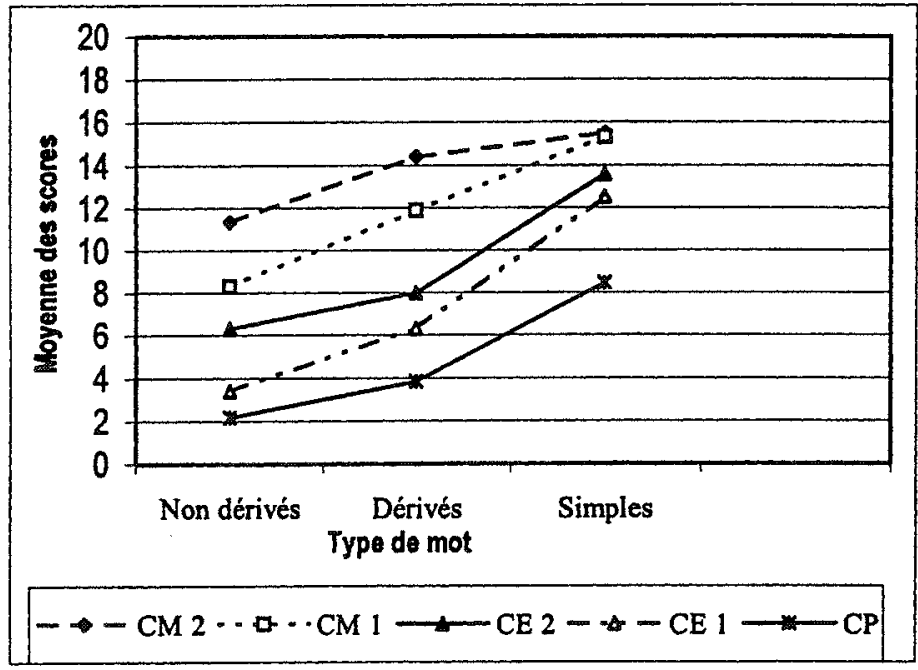

Figure 3 - Effet de la dérivation morphologique et du niveau scolaire sur le nombre d'items correctement orthographiés en ce qui concerne la résolution des difficultés orthographiques

Pour l'ensemble des sujets, l'analyse de variance conduite montre que les variables «type de mots» et «niveaux scolaires " ont un effet significatif à $p<0,001$ sur le score des mots correctement orthographiés, et que l'interaction des 2 variables est significative à $\mathrm{p}<0,001$ (les scores des mots «simples» sont relativement meilleurs chez les plus jeunes). Les différences entre les mots (non dérivés/dérivés, dérivés/simples, non dérivés/simples), et pour chacun des niveaux, sont significatives ( $\mathrm{p}<0,001 ; \mathrm{t}$ de Student cf. note 5 - pour échantillons appareillés). Les enfants dès le $\mathrm{CP}$ semblent donc capables de retrouver les mots référents pour orthographier les mots morphologiquement dérivés. On 
peut se demander malgré tout si la récupération des mots dérivés s'effectue bien par un transfert morphologique, ou plus exactement si les enfants n'ont pas simplement retrouvé les mots dérivés dans leur lexique mental, c'est-à-dire par la procédure d'adressage? L'épreuve 2 tente de lever cette ambiguîté.

\section{Epreuve 2}

Nous avons proposé aux sujets une épreuve de transcription de pseudo-mots construits à partir des mots référents et insérés soit dans des phrases sémantiquement congruentes (« Les pirates ont décidé le reterrement du trésor»), soit dans des phrases non congruentes (« Le spectacle est beau; c'est un vrai reterrement»). Ces pseudo-mots ne peuvent appartenir au lexique mental ; les sujets doivent donc utiliser pour les orthographier soit la voie phonologique (correspondances phonographémiques), soit une procédure analogique, soit avoir recours à leurs connaissances morphologiques. La proposition d'un contexte phrastique congruent devrait conduire les élèves à s'appuyer sur les mots référents.

La population expérimentale est la même que dans l'épreuve 1, à l'exception du niveau CP qui n'était pas encore suffisamment expert pour réaliser cette tâche.

\section{Hypothèse}

H5. Un pseudo-mot construit à partir d'une racine lexicale de la langue et possédant la difficulté orthographique présente dans cette racine devrait faire l'objet, en situation de phrases sémantiquement congruentes, d'un transfert morphologique pour que cette difficulté soit résolue.

\section{Plan d'expérience}

Nous avons 4 groupes indépendants correspondant aux 4 niveaux scolaires $(\mathrm{N})$, et qui sont confrontés aux 2 types de phrases, congruentes ou non (C), soit: $\underline{S}<N_{4}>{ }^{*} C_{2}$. 


\section{Résultats}

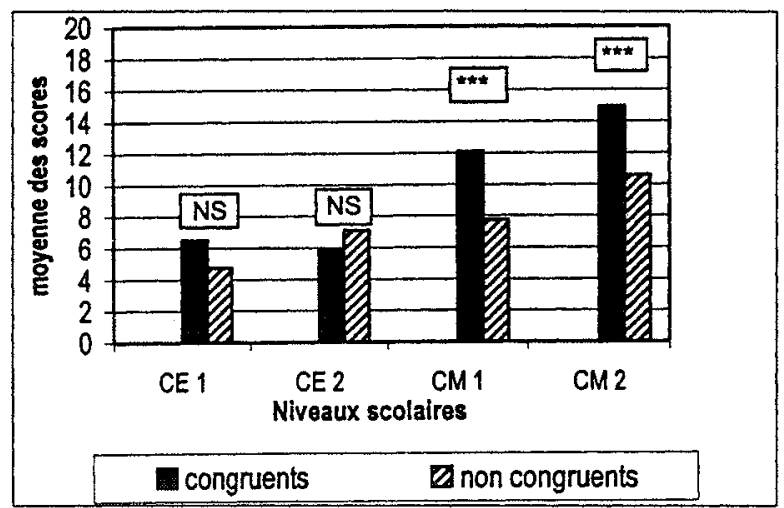

Figure 4 - Nombre de pseudo-mots écrits conformément aux dérivations supposées, en fonction de la congruence sémantique de la phrase et du niveau scolaire

Les différences de performances entre pseudo-mots congruents et non-congruents ne sont significatives ( $\mathrm{p}<0,001 ; \mathrm{t}$ de Student pour groupes indépendants) qu'à partir du niveau CM1. Comment expliquer cette absence de résultats pour les niveaux des CE 1 et 2 ? On peut faire deux hypothèses. La première tient compte de la grande perplexité chez les enfants les plus jeunes quant à «ces mots qui n'existent pas », et beaucoup d'entre eux ont souligné qu'il n'y avait pas de bonne orthographe pour des «faux» mots ${ }^{6}$. Ils ont donc pu employer préférentiellement les graphonèmes les plus fréquents quel que soit le contexte sémantique. On peut émettre une seconde hypothèse: dans la première épreuve, les mots à orthographier étaient connus oralement des enfants, la tâche en était donc facilitée et le recours à la morphologie pouvait être relativement automatique (activité «épilinguistique»), alors que les non-mots proposés dans la deuxième épreuve, par définition inconnus, nécessitaient une recherche

6. La consigne pour cette épreuve était la suivante: "Les mots à écrire sont dans une langue extra-terrestre; il faut écrire les mots le mieux possible, avec l'orthographe que vous pensez être la meilleure». 
consciente d'une solution orthographique (activité «métalinguistique »).

\section{Épreuve de contrôle}

Une épreuve "d'induction» a été proposée, et ce uniquement aux élèves de $\mathrm{CP}$ et $\mathrm{CE} 1$ pour lesquels on peut constater dans la première épreuve (cf. figure 3 ) des scores relativement faibles. Les mots dérivés sont présentés dans de nouvelles phrases mais qui comportent les mots référents en début de phrase (par exemple «Des petites bottes s'appellent des bottines»). Cette épreuve a été passée 10 jours après les premières pour éviter les effets d'accoutumance. Les scores des mots induits sont comparés dans la figure 5 ci-après aux résultats de l'épreuve 1.

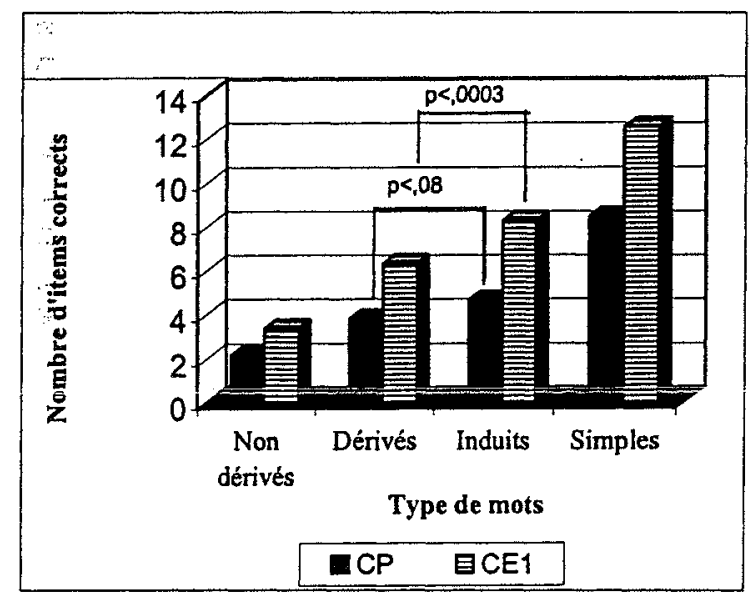

Figure 5 - Effet de la présence des mots référents en début de phrases et du niveau scolaire sur le nombre d'items cibles correctement orthographiés en ce qui concerne la résolution des difficultés orthographiques.

La différence entre mots dérivés non-induits et induits, déjà notable chez les CP ( $p<0,08 ; t$ de Student pour échantillons appareillés), est très significative pour les CE1 ( $p<0,0003$ ). Le fait d'induire explicitement la dérivation morphologique en proposant le mot référent en amorce à l'intérieur même de la phrase améliore les performances, et ce dès le début de l'apprentissage de l'orthographe. 
Nos résultats montrent bien l'utilisation, dès le niveau $\mathrm{CP}$ et de façon très importante au cycle 3 , de connaissances sur les liens morphologiques pour retrouver l'orthographe des mots. La comparaison des performances aux 3 épreuves de l'expérience II suggère que ces connaissances sont de type «épilinguistique », présentes mais non explicites avant le CM1, et que leur utilisation devient plus consciente à partir de ce niveau de développement. Ce type d'évolution a déjà été mis en évidence pour les autres capacités métalinguistiques: les jeunes enfants peuvent «jouer» avec les rimes (les comptines de l'école maternelle en sont un bon exemple) ou les syllabes des mots (les langues «enfantines» ou de certains groupes d'adolescents) sans pour autant comprendre les principes de la segmentation syllabique et encore moins phonémique. Or un certain nombre d'expériences d'entrainement métalinguistique, centrées sur les capacités segmentales mais aussi sur les caractéristiques du langage oral (traité comme objet à manipuler) et sur ses liens avec l'écrit, ont montré une amélioration notable des enfants entrainés dans leurs performances ultérieures en lecture (Ball et Blachman, 1991; Lecocq, 1991 ; Bruckert et Sanguin-Bruckert, 1993). Il n'est donc pas inconcevable de penser qu'un apprentissage plus systématique des composantes morphologiques de la langue par des exercices systématiques sur les «familles de mots» ou sur les passages entre féminin et masculin (et ce dans le cadre d'une réflexion plus générale sur les connaissances métalinguistiques), outre le fait d'enrichir les connaissances lexicales, permettrait d'accélérer la prise de conscience de la structure morphologique de ces mots. Le recours à ce type d'indices pourrait pallier les lacunes du lexique orthographique défaillant ou imprécis des enfants les moins compétents et contribuerait ainsi à lever le sentiment d'arbitrarité lié à un système graphique qui semble principalement soumis aux lois du hasard. 


\section{Bibliographie}

Alégria, J. et Mousty, P. (1994): Sur le développement de l'utilisation de certains aspects de la morphologie dans l'écriture du français, Actes du Ilème Congrès européen du Comité Permanent de Liaison des Orthophonistes et Logopèdes de la CEE, Anvers, NL.

AlÉGRIA, J. et Mousty, P. (1997): Processus lexicaux impliqués dans l'orthographe d'enfants francophones présentant des troubles de la lecture, in L. Rieben, M. Fayol et C.A. Perfetti (dir.), Des orthographes et leur acquisition, Lausanne, Delachaux et Niestlé, 167-180.

Ball, E.W. et Blachman, B.A. (1991): Dos phoneme awareness training in kindergarten make a difference in early word recognition and developmental spelling? Reading Research Quaterly, 26, 49-96.

Bosman, A.M. et Van Orden, G. (1997): Pourquoi l'orthographe est-elle plus difficile que la lecture? in L. Rieben, M. Fayol et C.A. Perfetti (dir.), Des orthographes et leur acquisition, Lausanne, Delachaux et Niestlé, 207-230.

Bruckert, J.-P. et Sanguin-Bruckert, Ch. (1993): Apprenti-lecteur, apprenti-linguiste? Le Gré des Langues, $5,141-157$.

Сатасн, N. (1980): L'orthographe française. Traité théorique et pratique, Paris, Nathan.

CATACH, N. (1988): L'écriture en tant que Plurisystème, ou Théorie de L. Prime, in N. Catach. (dir.), Pour une théorie de la langue écrite, Paris, Editions du CNRS, 243-259.

Chevrot, J.-P. (1996): La connaissance phonographique, Lidil, 13, 5-19.

EHRI, L.C. (1986): Sources of difficulty in learning to spell and read, in M. Wolraich et D. Routh (dir.), Advances in developmental and behavioral Pediatrics, vol 7, Greenwich, CT, Jai Press, 121-195.

FERreiro, E., Gomez-PALACIO, M. et al. (1988): Lire - Écrire à l'école: comment s'y apprennent-ils ? Lyon, CRDP.

GOMBERT, J.-E., BRYANT, P. et WARrICK, N. (1997): Les analogies dans l'apprentissage de la lecture et de l'orthographe, in L. Rieben, M. Fayol et C.A. Perfetti (dir.), Des 
orthographes et leur acquisition, Lausanne, Delachaux et Niestlé, 319-334.

HENDERSON, E. (1985): Teaching spelling, Boston, Houghton Mifflin.

LECOCQ, P. (1991): Apprentissage de la lecture et dyslexie, Bruxelles, Mardaga.

Millet, A. (1996): Orthographe française et phonographie, Lidil, 13, 21-40.

Mousty, P. et Alegria, J. (1996): L'acquisition de l'orthographe et ses troubles, in S. Carbonnel, P. Gillet, M.-D. Martory et S. Valdois (dir.), Approche cognitive des troubles de la lecture et de l'écriture chez l'enfant et l'adulte, Marseille, Solal.

Mousty, P. et Alegria, J. (1999): L'acquisition de l'orthographe: données comparatives entre enfants normo-lecteurs et dyslexiques, Revue française de pédagogie, 126, 7-22.

PeRfeTti, C.A. (1997): Psycholinguistique de l'orthographe et de la lecture, in L. Rieben, M. Fayol et C.A. Perfetti (dir), Des orthographes et leur acquisition, Lausanne, Delachaux et Niestlé, 37-56.

SANGUIN-BruCKerT, CH. et BruCKERT, J.-P. (2001): Les stratégies d'identification des mots dans l'apprentissage de la langue écrite, Revue de psychologie de l'éducation, 4, 123.

SANGUin-Bruckert, Ch. et Bruckert, J.-P. (2003): Les activités métalinguistique et mếtacognitive dans l'apprentissage de la lecture: le rôle de l'école, in M.N. Romdhane, J.-E. Gombert et M. Belajouza (dir.), L'apprentissage de la lecture. Perspectives comparatives, Rennes et Tunis, P.U. et CPU, 267-280.

SEYMOUR, P.H.K. (1997): Les fondations du développement orthographique et morphographique, in L. Rieben, M. Fayol et C.A. Perfetti (dir.), Des orthographes et leur acquisition, Lausanne, Delachaux et Niestlé, 385-403.

SPRENGER-Charolles, L. et CASAlis, S. (1996) : Lire. Lecture et écriture: acquisition et troubles du développement, Paris, PUF.

Thévenin, M.-G., Totereau, C., Fayol, M. et Jarousse, J.P. (1999): L'apprentissage/enseignement de la morpholo- 
gie écrite du nombre en français, Revue française de pédagogie, 126, 39-52.

Totereau, C., Thévenin, M.-G. et Fayol, M. (1997) : Acquisition de la morphologie du nombre à l'écrit en français, in L. Rieben, M. Fayol et C.A. Perfetti (dir.), Des orthographes et leur acquisition, Lausanne, Delachaux et Niestlé, 146-165.

Treiman, R. et Cassar, M. (1997): L'acquisition de l'orthographe en anglais, in L. Rieben, M. Fayol et C.A. Perfetti (dir.), Des orthographes et leur acquisition, Lausanne, Delachaux et Niestlé, 79-100. 